\title{
Acquired resistance of leukemic cells to AraC is associated with the upregulation of aldehyde dehydrogenase 1 family member A2.
}

$\operatorname{AUTHOR}(\mathrm{S})$ :

Kawasoe, Misaki; Yamamoto, Yasuko; Okawa, Katsuya; Funato, Tadao; Takeda, Mayu; Hara, Takeshi; Tsurumi, Hisashi; ... Matsunami, Hidetoshi; Markey, Sanford P; Saito, Kuniaki

\section{CITATION:}

Kawasoe, Misaki ... [et al]. Acquired resistance of leukemic cells to AraC is associated with the upregulation of aldehyde dehydrogenase 1 family member A2.. Experimental hematology 2013, 41(7): 597-603.e2

\section{ISSUE DATE:}

2013-07

\section{URL:}

http://hdl.handle.net/2433/176355

\section{RIGHT:}

(C) 2013 ISEH - Society for Hematology and Stem Cells. Published by Elsevier Inc.; This is not the published version. Please cite only the published version.; この論文は出版社版でありません。引用の際には 出版社版をご確認ご利用ください。 
Acquired resistance of leukemic cells to $\mathrm{AraC}$ is associated with the upregulation of aldehyde dehydrogenase 1 family member A2

Misaki Kawasoe $^{\mathrm{a} *}$, Yasuko Yamamoto ${ }^{\mathrm{a}}$, Tadao Funato ${ }^{\mathrm{b}}$, Mayu Takeda ${ }^{\mathrm{b}}$, Katsuya Okawa $^{\mathrm{c}}$, Takeshi Hara ${ }^{\mathrm{d}}$, Hisashi Tsurumi ${ }^{\mathrm{d}}$, Hisataka Moriwaki ${ }^{\mathrm{d}}$, Yuko Arioka ${ }^{\mathrm{a}}$, Masao Takemura $^{\mathrm{a}}$, Hidetoshi Matsunami ${ }^{\mathrm{e}}$, Sanford P. Markey ${ }^{\mathrm{f}}$, Kuniaki Saito ${ }^{\mathrm{a}}$

${ }^{a}$ Human Health Sciences, Graduate School of Medicine and Faculty of Medicine, Kyoto University, Japan

${ }^{\mathrm{b}}$ Department of Healthcare Economics and Quality Management, Tohoku Fukushi University, Japan

${ }^{\mathrm{c}}$ Drug Discovery Research Laboratories, Kyowa Hakko Kirin Co., Ltd., Shizuoka, Japan ${ }^{\mathrm{d}}$ First Department of Internal Medicine, Gifu University Graduate School of Medicine, Gifu, Japan

eMatsunami General Hospital, Kasamatsu, Gifu 501-6062, Japan

${ }^{\mathrm{f}}$ Laboratory of Neurotoxicology, National Institute of Mental Health, Bethesda, MD, USA

*These authors contributed equally to this work. 
Corresponding author: Yasuko Yamamoto Ph.D., Human Health Sciences, Graduate School of Medicine and Faculty of Medicine, Kyoto University, Kyoto 606-8507, Japan

Tel/Fax: +81-75-751-3957

E-mail: yamamoto-y@hs.med.kyoto-u.ac.jp

Abbreviation: AraC, 1- $\beta$-D-arabinofuranosylcytosine; AML, acute myeloid leukemia; ALDH1A2, aldehyde dehydrogenase 1 family member A2.

Key words: drug resistance, AraC, ALDH1A2 


\section{Abstract}

The elucidation of drug resistance mechanisms is very important in the development of clinical therapies for the treatment of leukemia. To study the drug resistance mechanisms, protein expression profiles of $1-\beta-\mathrm{D}$-arabinofuranosylcytosine (AraC) sensitive K562 (K562S) cells and AraC resistant K562 (K562AC) cells were compared using two-dimensional fluorescence difference gel electrophoresis. In comparison of protein expression profiles, 2073 protein spots were found to be altered, 15 proteins of them were remarkably altered. These proteins were identified by mass spectrometry. The most differently expressed proteins were aldehyde dehydrogenase 1 family member A2 (ALDH1 A2) and vimentin. Both proteins were verified using reverse transcriptase polymerase chain reaction (RT-PCR) and Western blot analysis. ALDH1A2 protein was found to be effective in AraC resistance. ALDH1A2 knock down (KD) induced sensitivity to AraC treatment in K562AC cells, and ALDH1A2 overexpressed K562S cells acquired the AraC resistance. Further, the findings also suggest that ALDH1A2 expression is increased after the appearance of AraC resistance in clinical cases. These results will be helpful in understanding the mechanism of AraC resistance. 
Introduction

Chemotherapy has been very useful in the treatment of leukemia. However, cellular drug resistance is the greatest impediment to successful treatment. The mechanism of drug resistance can roughly be divided into two processes: drug uptake or metabolism, and inhibition of apoptotic cell death or increased repair of drug-induced DNA damage $[1,2]$.

Cytosine arabinoside (1- $\beta$-D-arabinofuranosylcytosine, AraC) is one of the most effective drugs in the chemotherapeutic treatment of acute myeloid leukemia (AML) [3]. AraC is also active against other hematologic malignancies such as acute lymphoblastic leukemia (ALL) and non-Hodgkin's lymphoma [4]. Laboratory investigations to elucidate the biochemical correlation between drug action and drug resistance mechanisms have extensively examined the clinical importance of $\mathrm{AraC}$, in order to obtain leads for the development of better therapeutic strategies.

AraC is converted into its active metabolite, cytosine arabinoside triphosphate (Ara-CTP), intracellularly in leukemic blasts by deoxycytidine kinase (dCK) [5]. Ara-CTP is generally considered to be the active form of the drug since it both inhibits DNA polymerase and leads to AraC incorporation into DNA [6]. AraC is catabolized to 
the non-toxic metabolite, arabinosideuridine (AraU), by a rapid deamination mediated by cytidine deaminase (CDD) [7, 8]. Various biochemical mechanisms of AraC resistance have been shown in experimental animal tumor models or in vitro systems [9]. Low AraC phosphorylation or low dCK in marrow blasts has been associated with poor response [10]. It has also been reported that AraC resistance is induced when the influx of $\mathrm{AraC}$ is reduced because of the human equilibrative nucleoside transporter 1 (hENT1) [11], when the degradation of AraC is enhanced or when there is excessive conversion of AraC to inactive AraU due to high levels of CDD [12]. Although multidrug resistance (MDR1) gene and multidrug resistance-associated protein (MRP) gene are commonly involved in drug resistance, both the genes have not been observed in AraC resistant cells [13]. Various studies have been performed to elucidate the mechanism of AraC resistance, but it still remains unexplained.

Therefore, the present study aimed at investigating the expression of proteins associated with the acquisition of resistance to AraC. The expression of the proteins was examined using two-dimensional fluorescence difference gel electrophoresis (2D-DIGE) in AraC-sensitive (K562S) and AraC-resistant (K562AC) cell lines derived from K562. 
Materials and Methods

\section{Cell Culture}

Human leukemia cell lines, K562S and K562AC, were obtained from the School of Medicine, Tohoku University. The resistant clones, termed K562/AC, were established and grown for 3 months, during which they were continuously exposed to increasing concentrations of Ara-C up to $50 \mathrm{mM}$, and were maintained in medium containing Ara-C. K562AC cells were established before, as described by Funato et al [13]. K562S cells were confirmed to be sensitive to AraC and K562AC cells were confirmed to be resistant to Ara-C (Nihon Shinyaku Co., Japan). The cells were grown in RPMI-1640 medium (Nissui Pharmaceutical Co., Japan) with 10\% fetal calf serum (Cellect, $\mathrm{OH}, \mathrm{USA}$ ) at $37^{\circ} \mathrm{C}$ in $5 \% \mathrm{CO}_{2}$. Doxorubicin resistant derivative of $\mathrm{K} 562$ cell line was obtained from JCRB Japan, and maintained in medium containing Doxorubicin (Wako Co., Japan).

Cell Viability Assay

Cell viability was determined by the MTT assay method, using CellTiter 96 AQueous Cell Proliferation Assay (Promega, Madison, USA). A total of $10^{4}$ cell lines were plated in each well of a 48-well tissue culture plate. At the indicated times, cell viability was 
measured. Untreated cells served as control. The viability of treated cells was calculated as a percentage of viability relative to the untreated control cells.

\section{D-DIGE}

Cell pellets were dissolved in lysis buffer (7M urea, $2 \mathrm{M}$ thiourea, 1\% Triton X-100, 4\%

CHAPS, and $10 \mathrm{mM}$ Tris). Cell lysates were labeled with $\mathrm{Cy} 2, \mathrm{Cy} 3$, and $\mathrm{Cy} 5$, according to the protocols described in the Ettan DIGE user manual (18-1164-40 Edition AA, GE Healthcare). The 2 Dimensional Fluorescence Difference Gel Electrophoresis (DIGE) was performed according to the method previously reported [14] with some modifications. IPG strips (24 cm, pH 3-10 nonlinear, GE) and Ettan IPGphor System (GE Healthcare, Uppsala, Sweden) were used for the first-dimension IEF. After 2-DE, gels were scanned on Typhoon 9410 scanner (GE Healthcare, Uppsala, Sweden) using the Ettan DALT gel alignment guides.

Gel Imaging and Statistical Analysis

Gel images were cropped using ImageQuant TL 2003 (GE Healthcare, Uppsala, Sweden) and spot detection was performed with DeCyder 6.5 DIA (Difference In-gel Analysis) software (GE Healthcare, Uppsala, Sweden). The gel images were matched using DeCyder 6.5 BVA (Biological Variation Analysis) software (GE Healthcare, Uppsala, Sweden). Statistical analysis was performed using paired Student's t-test with 
false discovery rate correction using DeCyder 6.5 BVA. A p<0.05 was considered statistically significant.

For protein identification, individual spots were digested with trypsin. Molecular mass analyses of triptic peptides were performed by matrix-assisted laser desorption/ionization time-of-flight mass spectrometry (MALDI-TOF/MS) using an ultraflex TOF/TOF (Bruker Daltonics). Proteins were identified by comparison between the molecular weights determined by MALDI-TOF/MS and theoretical peptide masses from the proteins registered in NCBInr [15].

RNA Extraction and semi-quantitative analysis of RT-PCR products

Total RNA was isolated from cell lines with Isogen (Nippon Gene, Tokyo, Japan) and the RNA concentration was determined spectrophotometrically at $260 \mathrm{~nm}$. Reverse transcription-PCR was performed using ReverTra Ace qPCR RT Kit (Toyobo, Tokyo, Japan). The following PCR primers were synthesized:

Vimentin forward: 5'-TGTTGACAATGCGTCTCTGGCAC-3, reverse: 5'-ACTCCAGTCCGAACCTTTGTAGG-3', ALDH1A2 forward: 5'-AGTGTGGAGAAGGATGGATG-3', reverse: 5'-AGGTGTCGTATCAGGTTCAG-3', 
GAPDH forward: 5'-GCCAAGGTCATCCATGACAAC-3', reverse: 5'-AGTGTAGCCCAAGATGCCCTT-3'. Semi-quantitative analysis of RT-PCR products was performed by using NIH ImageJ $1.34 \mathrm{~s}$ software and normalized to GAPDH. Shown were the results of one of three expresiments with similar results.

\section{Western Blot}

Cell extracts were prepared in lysis buffer [100 mM Tris-HCL (pH 8.0), $100 \mathrm{mM} \mathrm{NaCl}$, $0.5 \% \mathrm{NP} 40$, and $0.2 \mathrm{mM}$ phenylmethanesulfonyl fluoride], and centrifuged for five minutes at $1800 \mathrm{x} g$ at $4^{\circ} \mathrm{C}$. The supernatant was collected for protein analysis. Proteins in the supernatant were lysed with SDS-PAGE sample buffer, sonicated, and boiled. The protein samples were then separated by one-dimensional SDS-PAGE, transferred onto a PVDF membrane, and blotted with the appropriate antibodies. The immunoblot signals were normalized by the corresponding $\beta$-actin signal. Antibodies used for Western blot analysis were anti-vimentin polyclonal antibody (pAb), anti-ALDH1A2 monoclonal antibody (mAb), and anti-GAPDH pAb. Anti-vimentin pAb and anti-ALDH1A2 mAb were obtained from Abcam (Cambridge, UK) and anti-GAPDH pAb was obtained from Santa Cruz Biotechnology (CA, USA).

ALDH1A2 constructs and gene transfection. 
We used RT-PCR to synthesize the full length cDNA for human ALDH1A2 (NM_170696). Total RNA was extracted from K562AC cells and used for the synthesis of the cDNAs. Full-length human ALDH1A2 cDNAs were subcloned into the N-terminus venus-tagged CAGGS expression vector. ALDH1A2 gene transfection to K562S cells was performed using Lipofectamine Plus (Invitrogen, Carlsbad, CA), following the manufacturer's instructions. To establish stable transfectants, transfected cells were selected by the medium containing $500 \mu \mathrm{g} / \mathrm{ml} \mathrm{G} 418$, and clones derived from single cells were picked up.

RNA Interference Experiments Using Small Interfering RNAs (siRNAs)

ALDH1A2 siRNA-1 (s16907) was purchased from Ambion (Life Technologies, Japan). K562AC cells were transfected with siRNA using Lipofectamine Reagent (Life Technologies, Japan). As a negative control, a scrambled oligonucleotide, Negative control \#2 (Life Technologies, Japan), was used. At $48 \mathrm{~h}$ after transfection, cells were treated with AraC for $24 \mathrm{~h}$. Cell survival was determined using MTT assay, as described above.

Histopathology

Smeared bone marrow slides were fixed with $100 \%$ methanol. The slide was treated with $0.3 \%$ hydrogen peroxide to block nonspecific staining. The primary antibody used 
was rabbit anti-ALDH1A2 polyclonal antibody (Abcam, Cambridge, UK). The slide was incubated overnight with the antibody at $4^{\circ} \mathrm{C}$. After washing in $50 \mathrm{mM}$ Tris-buffered saline, a refined avidin-biotin technique in which a biotinylated secondary antibody reacts with several peroxidase-conjugated streptavidin molecules was employed for amplification using a DAKO LSAB+/HRP kit (Dako, Tokyo, Japan). Diaminobenzidine tetrahydrochloride (DAB) was used for the visualization of immunoreactive cells. The nuclei of cells were counterstained with hematoxylin. The total number of cells was counted; it was checked to determine whether the cells were positive or not. The ratio of positive cells to total cells was used to estimate the rate of ALDH1A2 positive cells.

\section{Clinical Case}

Peripheral blood was drawn from five patients with AraC resistance. The diagnosis of leukemia was based on clinical features and hematological characteristics. Ara-C resistance was clinically defined as follows: The case could not achieve complete remission (CR) by one or two courses of induction chemotherapy consisting of 12 $\mathrm{mg} / \mathrm{m}^{2}$ idarubicin [IDA] on days $1-3$ and $100 \mathrm{mg} / \mathrm{m}^{2}$ Ara-C on days $1-7$ and salvage chemotherapy with high-dose Ara-C (2000 mg/m² Ara-C every 12 hours on days 1-5). CR was defined according to Cheson's criteria [16]. Informed consent was obtained 
according to the Declaration of Helsinki. The study protocol comprised a retrospective case series design that was approved by the institutional review board at Gifu university hospital. Supplement Table 1 shows the main clinical findings of all the patients studied. Statistical Analysis

The results depicted in Fig. 3, Fig. 4 and Fig. 5 are expressed as means \pm SD. Statistically significant differences $(\mathrm{p}<0.05)$ between two groups were determined using Student's t-test. 
Results

The protein profiles by $2 D-D I G E$

Protein expression in K562S and K562AC cells was compared using 2D-DIGE. AraC-resistance in K562 cells was established before, as described previously [13]. To confirm the resistance or sensitivity to AraC in $\mathrm{K} 562 \mathrm{~S}$ and $\mathrm{K} 562 \mathrm{AC}$ cells, the viability of both cells after AraC treatment was examined using the MTT assay. After AraC treatment, there was no growth of $\mathrm{K} 562 \mathrm{~S}$ cells, while the proliferation of K562AC cells was unaffected. (Supplement Fig. 1).

In order to identify the proteins involved in the acquisition of $\mathrm{AraC}$ resistance, the differences in protein expression in K562S and K562A were examined by DIGE and mass spectrometry. The total number of differentially expressed proteins identified in the two cell lines was 2073. Of the proteins identified, the differences in expression of 15 proteins were found to be statistically significant (Fig. 1). These proteins were identified by mass spectrometry. Table 1 shows a list of the differentially expressed proteins with their protein accession numbers, calculated fold changes, and p values. The 15 proteins were assigned to four categories based on their function, namely macromolecular metabolism (amino acid and sugar metabolism), molecular cytoskeleton reorganization, signal transduction, and oxidative stress. 
To validate the differential protein expression, RT-PCR and western blotting were performed for two proteins with the highest differential rate. The two proteins were found to be ALDH1A2 and vimentin (Fig. 2).

\section{Effects of ALDH1A2 on AraC resistance}

It has recently been reported that the amount of expressed ALDH isoform correlates with drug resistance [17] [18]. And, some reports showed that retinoic acid inhibited the vimentin expression [19]. We examined the expression of vimentin in K562S treated with retinoic acid. The retinoic acid treatment decreased the vimentin expression (data not shown). This result suggested that vimentin expression was controlled by ALDH1A2 expression. Thus, RALDH2 expression was considered to be more important to acquire the resistance than vimentin expression. Therefore, the effect of ALDH1A2 on AraC resistance in K562AC cells was investigated here. This was done by knocking down ALDH1A2 in K562AC cells using siRNA. ALDH1A2 knock down (KD) was confirmed using RT-PCR and western blotting (Fig. 3A). Cell viability was compared between the ALDH1A2 KD cells treated with and without AraC. The viability of ALDH1A2 KD cells was found to be reduced by AraC treatment. This shows that ALDH1A2 KD induces sensitivity to AraC in K562AC cells (Fig. 3B). To confirm the effect of ALDH1A2 on AraC resistance, the ALDH1A2 gene was 
transfected to K562AS cells. The cells with this transfection acquired the resistance to $\operatorname{AraC~(Fig.~4).~}$

Immunohistochemical analysis of ALDH1A2 in AraC-Resistant Patients

An in vitro study has suggested that ALDH1A2 may be associated with AraC resistance.

Therefore, the expression of ALDH1A2 in the peripheral blood of AraC-resistant patients was examined using immunohistochemical analysis. ALDH1A2 expression in AraC-resistant patients was compared before and after the acquisition of resistance. Fig. 5 shows that ALDH1A2 expression was high in patients with AraC resistance and that all patients had increased ALDH1A2 expression after the acquisition of AraC resistance. 
Discussion

The present study reveals that 15 proteins, including ALDH1A2, present in K562AC cells are significantly down- or up-regulated. ALDH1A2 belongs to the aldehyde dehydrogenase (ALDH) family. Twelve ALDH genes have been identified in humans. These genes, located on different chromosomes, encode a group of enzymes which oxidize a variety of aliphatic and aromatic aldehydes [20,21]. Many studies have shown that some ALDH family members play an important role in drug resistance $[17,18]$. ALDH3A1 (a member of the ALDH family) expression in tumor cells has been shown to induce resistance to drug toxicity, resulting in a poor response to anti-tumor drugs. As previously reported, ALDH3A1 is associated with resistance to etoposide and Mitomycin C [18]. It is also known that transfection with rat ALDH3 confers oxazaphosphorine-specific resistance on human MCF-7 cells. The resistance is also known to be reversed upon pretreatment with diethylaminobenzaldehyde, an ALDH3 inhibitor [22]. It has also been previously reported that overexpression of ALDH1A2 induces drug resistance [17].

ALDH1A2 is an enzyme that catalyzes the synthesis of retinoic acid from retinaldehyde. Retinoic acid is an active metabolite of vitamin A and regulates a wide range of biological processes including cell proliferation, differentiation, and 
morphogenesis [23]. Recently, Moreb et al showed that the overexpression of both ALDH1A2 and ALDH2 results in increased relative resistance to both 4-Hydroperoxycyclophosphamide (4-HC) and doxorubicin, but resistant mechanisms did not reveled [17]. We also examined the protein expression pattern in K562 doxorubicin resistant cells using 2D-DIGE. 16 proteins were remarkably altered between K562 doxorubicin and K562S (Supplemental figure. 2). Our results showed that ALDH1A2 expression did not altered in K562 cell with doxorubicin resistance. The identified proteins were different between AraC resistance cells and doxorubicin resistance cells. These protein profiles revealed the participation of different proteins in the resistant mechanism of each two drugs.

The results of the present study using K562AC cells suggest that the expression of ALDH1A2 increases in AraC-resistant cells. Furthermore, ALDH1A2 KD is found to induce sensitivity to AraC treatment in K562AC cells, and ALDH1A2 overexpression on $\mathrm{K} 562 \mathrm{~S}$ cells induced the AraC resistance. It is speculated that ALDH1 A2 expression in $\mathrm{K} 562 \mathrm{AC}$ cells induces the accumulation of RA in the cytosolic space. Retinoic acid accumulation may be involved in the anti-apoptotic property. Kitamura et al. have shown that retinoic acid plays a role in the signal transduction pathways involved in hydrogen peroxide $\left(\mathrm{H}_{2} \mathrm{O}_{2}\right)$-induced apoptosis [24]. $\mathrm{H}_{2} \mathrm{O}_{2}$ induces apoptosis in mesangial 
cells via activation of c-Jun-N-terminal kinase (JNK) and extracellular signal-regulated kinase (ERK). ERK and JNK induce accumulation and activation of activator protein-1 (AP-1), causing apoptosis. RA inhibits the apoptotic process by suppressing AP-1. This is because retinoic acid attenuates the expression of c-Fos/c-Jun and activation of JNK, at least in part, via induction of the dual-specificity protein phosphatase, MAP kinase phosphatase 1 (MKP-1) [25]. These findings suggest that ALDH1A2 may contribute to protection of drug-resistant cells from the apoptotic signaling by $\mathrm{AraC}$ in $\mathrm{K} 562$ cells.

Further studies are still needed to verify the possible mechanisms for AraC resistance. However, the results of the present study provide useful insights into the mechanisms of drug resistance and allow us to assume that various molecules participate in the mechanism. It is difficult to elucidate the actual mechanism of drug resistance because of the up-regulation of only ALDH1A2, although it is known that ALDH1A2 plays an important role. Studying the interaction between ALDH1A2 and other molecules may be useful for the elucidation of the mechanism of AraC resistance.

\section{Acknowledgement}

This study was supported in part by a Grant-in-Aid from the Ministry of Education, Science and Culture of Japan (to K. Saito 23390149) and (to Y. Yamamoto 23790621). 
This work was also supported in part by a grant from the Smoking Research Foundation.

We thank Dr. Y. Ohta for scientific discussion and proofreading the manuscript.

\section{Conflict of Interest statement}

The authors declare that there are no conflicts of interest. 
References

[1] Kartalou M, Essigmann J M, Mechanisms of resistance to cisplatin. Mutation

Research-Fundamental and Molecular Mechanisms of Mutagenesis. 2001; 478: 23-43.

[2] Rabik C A, Dolan M E, Molecular mechanisms of resistance and toxicity associated with platinating agents. Cancer Treatment Reviews. 2007; 33: 9-23.

[3] Cunningham I, A basis for updating our approach to resistant acute leukemia.

American Journal of Hematology. 2012; 87: 251-257.

[4] Braess J, Freund M, Hanauske A et al, Oral cytarabine ocfosfate in acute myeloid leukemia and non-Hodgkin's lymphoma - phase I/II studies and pharmacokinetics.

Leukemia. 1998; 12: 1618-1626.

[5] Flasshove M, Strumberg D, Ayscue L et al, Structural-analysis of the deoxycytidine kinase gene in patients with acute myeloid-leukemia and resistance to cytosine-arabinoside. Leukemia. 1994; 8: 780-785.

[6] Abdel-Aziz W, Jiang H Y, Hickey R J, Malkas L H, Ara-C affects formation of cancer cell DNA synthesome replication intermediates. Cancer Chemotherapy and Pharmacology. 2000; 45: 312-319. 
[7] Abraham A, Varatharajan S, Abbas S et al, Cytidine deaminase genetic variants influence RNA expression and cytarabine cytotoxicity in acute myeloid leukemia. Pharmacogenomics. 2012; 13: 269-282.

[8] Laliberte J, Momparler R L, Human cytidine deaminase - purification of enzyme, cloning, and expression of its complementary-DNA. Cancer Research. 1994; 54: $5401-5407$

[9] Tallman M S, Gilliland D G, Rowe J M, Drug therapy for acute myeloid leukemia.

Blood. 2005; 106: 1154-1163.

[10] Popkov M, Lussier I, Medvedkine V et al., Multidrug-resistance drug-binding peptides generated by using a phage display library. European Journal of Biochemistry. 1998; 251: 155-163.

[11] White J C, Hines L H, Winters K L, Concentration-dependence of Cytosine-arabinoside (Ara-C) phosphorylation by human-leukemia cells and human Ara-C kinase. Proceedings of the American Association for Cancer Research. 1987; 28: $323-323$

[12] Jin G, Matsushita H, Asai S et al, FLT3-ITD induces ara-C resistance in myeloid leukemic cells through the repression of the ENT1 expression. Biochemical and Biophysical Research Communications. 2009; 390: 1001-1006. 
[13] Funato T, Satou J, Nishiyama Y et al., In vitro leukemia cell models of Ara-C resistance. Leukemia Research. 2000; 24: 535-541.

[14] Zhou G, Li H M, DeCamp D et al., 2D differential in-gel electrophoresis for the identification of esophageal scans cell cancer-specific protein markers. Molecular \& Cellular Proteomics. 2002; 1: 117-124.

[15] Jensen O N, Podtelejnikov A, Mann M, Delayed extraction improves specificity in database searches by matrix-assisted laser desorption/ionization peptide maps. Rapid Communications in Mass Spectrometry. 1996; 10: 1371-8.

[16] Cheson, B. D., Bennett, J. M., Kopecky, K. J., Buchner, T., et al., Revised recommendations of the international working group for diagnosis, standardization of response criteria, treatment outcomes, and reporting standards for therapeutic trials in acute myeloid leukemia. Journal of Clinical Oncology. 2003, 21, 4642-4649.

[17] Moreb J S, Ucar D, Han S et al, The enzymatic activity of human aldehyde dehydrogenases 1A2 and 2 (ALDH1A2 and ALDH2) is detected by Aldefluor, inhibited by diethylaminobenzaldehyde and has significant effects on cell proliferation and drug resistance. Chemico-Biological Interactions. 2012; 195: 52-60. 
[18] Pappa A, Brown D, Koutalos Y et al, Human aldehyde dehydrogenase 3A1 inhibits proliferation and promotes survival of human corneal epithelial cells. Journal of Biological Chemistry. 2005; 280: 27998-28006.

[19] Muzio G, Maggiora M, Paiuzzi E, Oraldi M, Canuto, R. A., Aldehyde dehydrogenases and cell proliferation. Free Radical Biology and Medicine. 2012; 52: 735-746.

[20] Yoon, W. H., Song, I. S., Lee, B. H., Jung, Y. J., et al., Differential regulation of vimentin mRNA by 12-O-tetradecanoylphorbol 13-acetate and all-trans-retinoic acid correlates with motility of Hep 3B human hepatocellular carcinoma cells. Cancer Letters. 2004, 203, 99-105.

[21] Yoshida A, Rzhetsky A, Hsu L C, Chang C, Human aldehyde dehydrogenase gene family. European Journal of Biochemistry. 1998; 251: 549-557.

[22] Sreerama L, Sladek N E, Cellular levels of class 1 and class 3 aldehyde dehydrogenases and certain other drug-metabolizing enzymes in human breast malignancies. Clinical Cancer Research. 1997; 3: 1901-1914.

[23] De Luca L M, Retinoids and their receptors in differentiation, embryogenesis, and neoplasia. Faseb Journal. 1991; 5: 2924-2933. 
[24] Kitamura M, Ishikawa Y, Moreno-Manzano V et al, Intervention by retinoic acid in oxidative stress-induced apoptosis. Nephrology Dialysis Transplantation. 2002; 17 : 84-87.

[25] Moreno-Manzano V, Ishikawa Y, Lucio-Cazana J, Kitamura M, Suppression of apoptosis by all-trans-retinoic acid - Dual intervention in the c-Jun N-terminal kinase-AP-1 pathway. Journal of Biological Chemistry. 1999; 274: 20251-20258. 
Figure captions

Figure 1.

2D-DIGE gel images of K562S labeled with Cy5 and K562AC labeled with Cy3. (A)

Spots of proteins differentially expressed $(\mathrm{p}<0.05$, fold change $\geq 2.0$ ) were selected for identification. (B) Spots 4, 5 and 6, examples evaluated using Decyder. The top panel is the partial view. The middle panel is the 3D image. The bottom panel analyzed to each spot about relative protein abundance (Standard, K562 and K562AC).

Figure 2.

RT-PCR and western blotting analysis of vimentin, ALDH1A2, and GAPDH in K562S and $\mathrm{K} 562 \mathrm{AC}$.

To validate the differing expressions of proteins in K562S and K562AC, expression of both ALDH1A2 and vimentin genes was observed in both cell lines. (A) RT-PCR showing the mRNA expressions of ALDH1A2 and vimentin in K562S and K562AC. (B) Western blots showing the expression level of ALDH1A2 and vimentin in K562S and $\mathrm{K} 562 \mathrm{AC}$.

Figure 3.

Effects of AraC treatment in K562AC with ALDH1A2 KD 
(A) ALDH1A2 KD in K562AC examined by RT-PCR and western blot. (B) Proliferation of K562AC with ALDH1A2 KD after AraC treatment examined using the proliferation assay. ALDH1A2 KD induced sensitivity to AraC. Data are shown as the mean \pm SD of three independent experiments. ${ }^{*} p<0.05$.

Figure 4.

Effects of ALDH1A2 overexpression in K562S.

(A) ALDH1A2 overexpression in K562S confirmed by western blot. (B) Proliferation of K562S with ALDH1A2 overexpressed after AraC treatment examined using the proliferation assay. ALDH1A2 overexpression induced resistance to AraC. Data are shown as the mean \pm SD of three independent experiments. ${ }^{*} \mathrm{p}<0.001$.

Figure 5.

Immunohistochemical analysis of ALDH1A2 in AraC resistant patients.

(A) ALDH1A2 expression observed in the peripheral blood of Ara-C-resistant patients before and after the acquisition of AraC resistance. The results of one of the five analyses are shown because similar results were obtained in the five analyses (a, c: lower magnification, $\mathrm{b}$, d: higher magnification). (B) Changes in ALDH1A2 expression 
in leukemia cells before and after the acquisition of $\mathrm{AraC}$ resistance. The rate of positive cells in all AraC resistant patients is summarized. $* \mathrm{p}<0.05$.

Supplement Figure 1.

Viability of K562S and K562AC cells after AraC treatment.

$\mathrm{K} 562 \mathrm{~S}$ and K562AC cells were treated in the presence or absence of AraC. After $24 \mathrm{~h}$, $48 \mathrm{~h}$, and $72 \mathrm{~h}$ of AraC treatment, proliferation was determined by the cell proliferation assay. The results are expressed as the mean \pm SD of three independent experiments.

Supplement Figure 2.

2D-DIGE gel images of K562S labeled with Cy5 and K562DOX labeled with Cy3. (A) Spots of proteins differentially expressed $(p<0.05$, fold change $\geq 2.0)$ were selected for identification. 
A
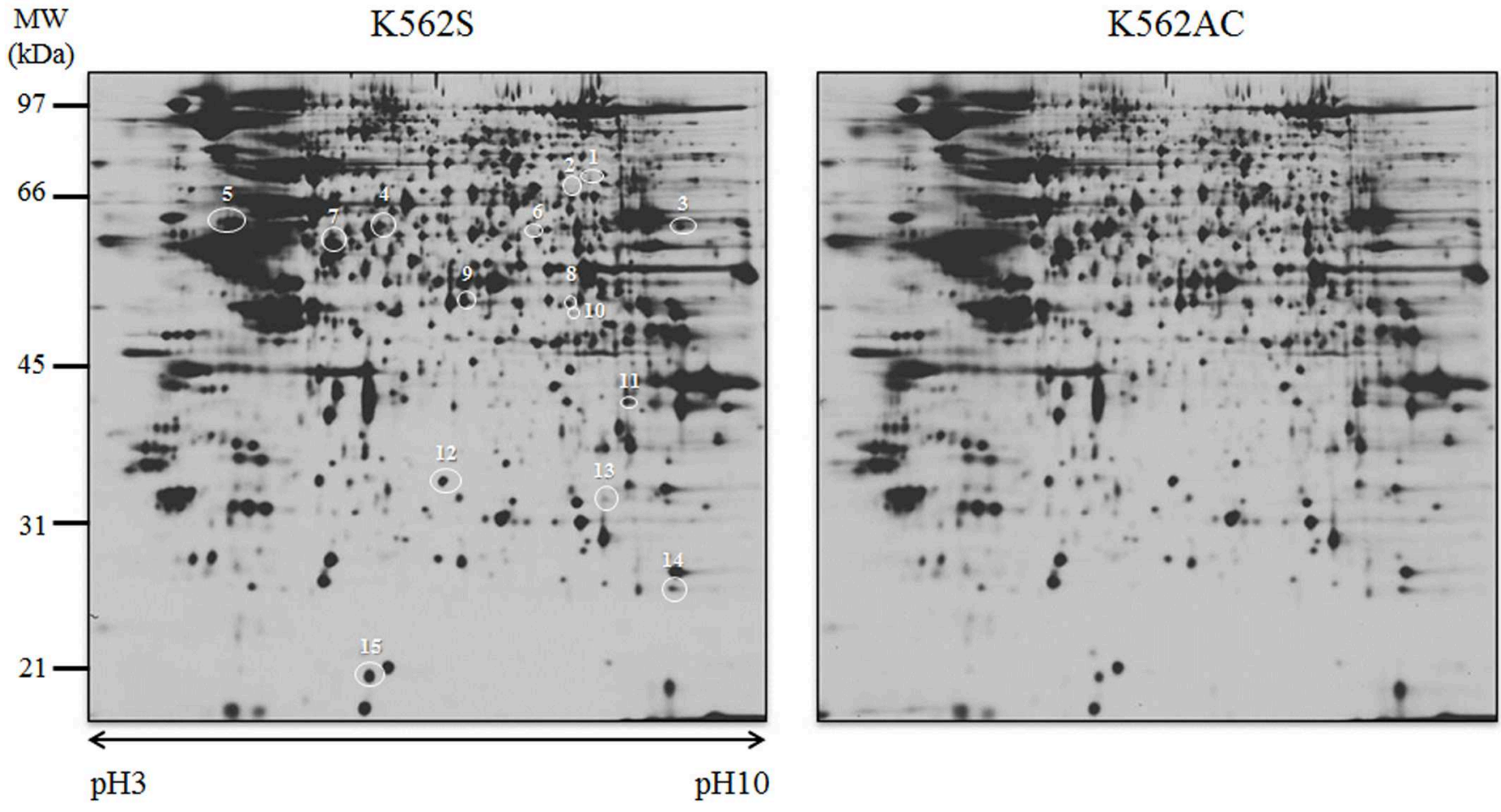

B

Spot 4

Spot 5

K562S

$\mathrm{K} 562 \mathrm{AC}$

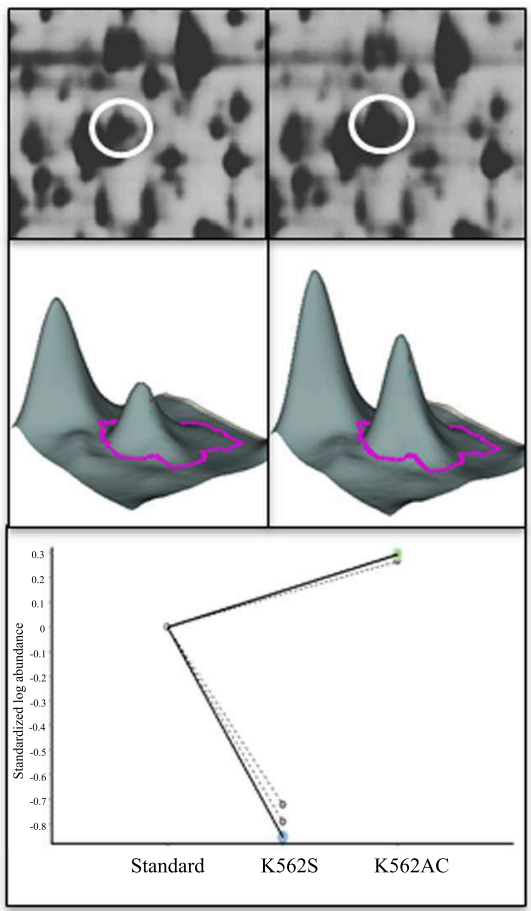

K562S

$\mathrm{K} 562 \mathrm{AC}$

K562S

Spot 6
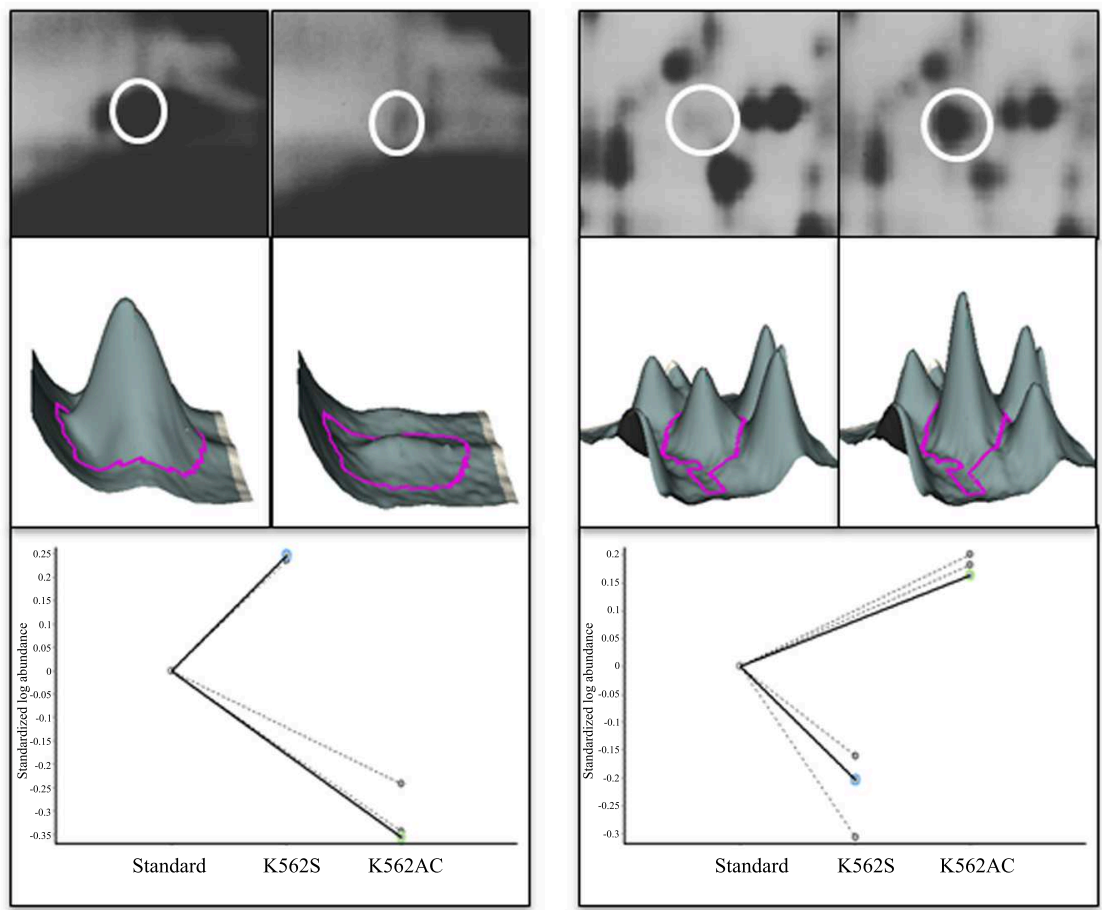

Figure 1. Kawasoe et al 
A

B

Vimentin

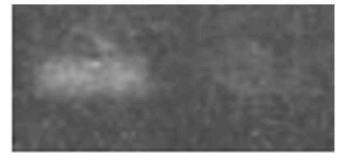

Vimentin

ALDH1A2

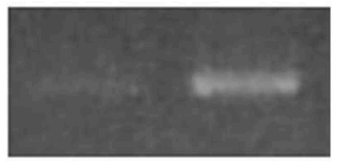

ALDH1A2

GAPDH

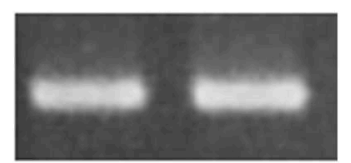

GAPDH

$\mathrm{S} \quad \mathrm{AC}$

Figure 2. Kawasoe et al 
A

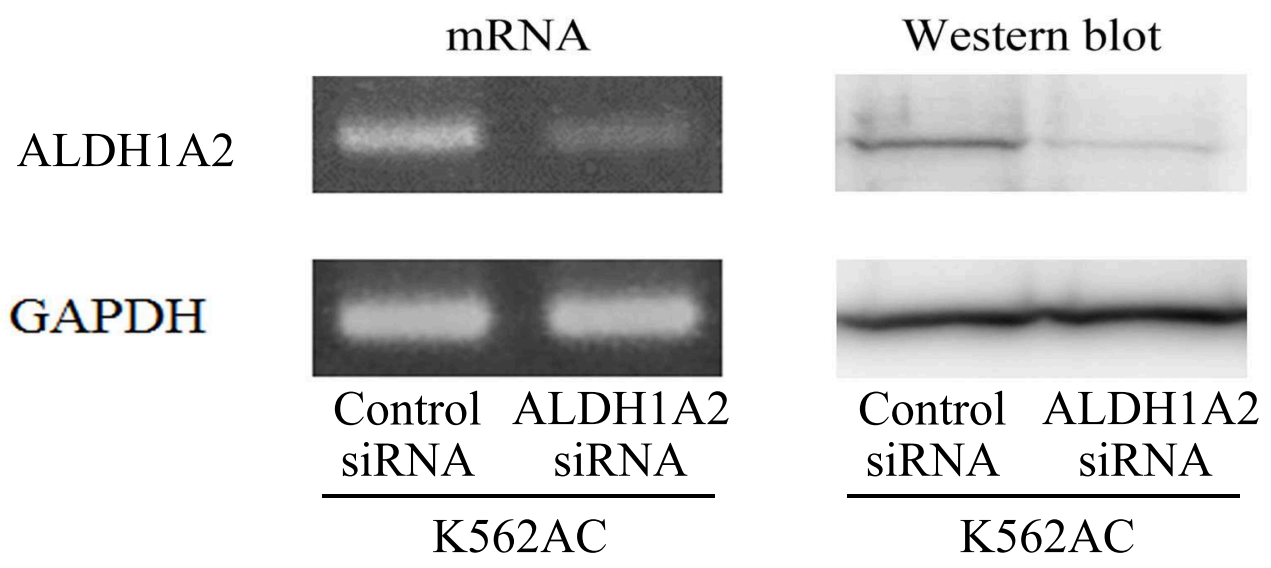

B

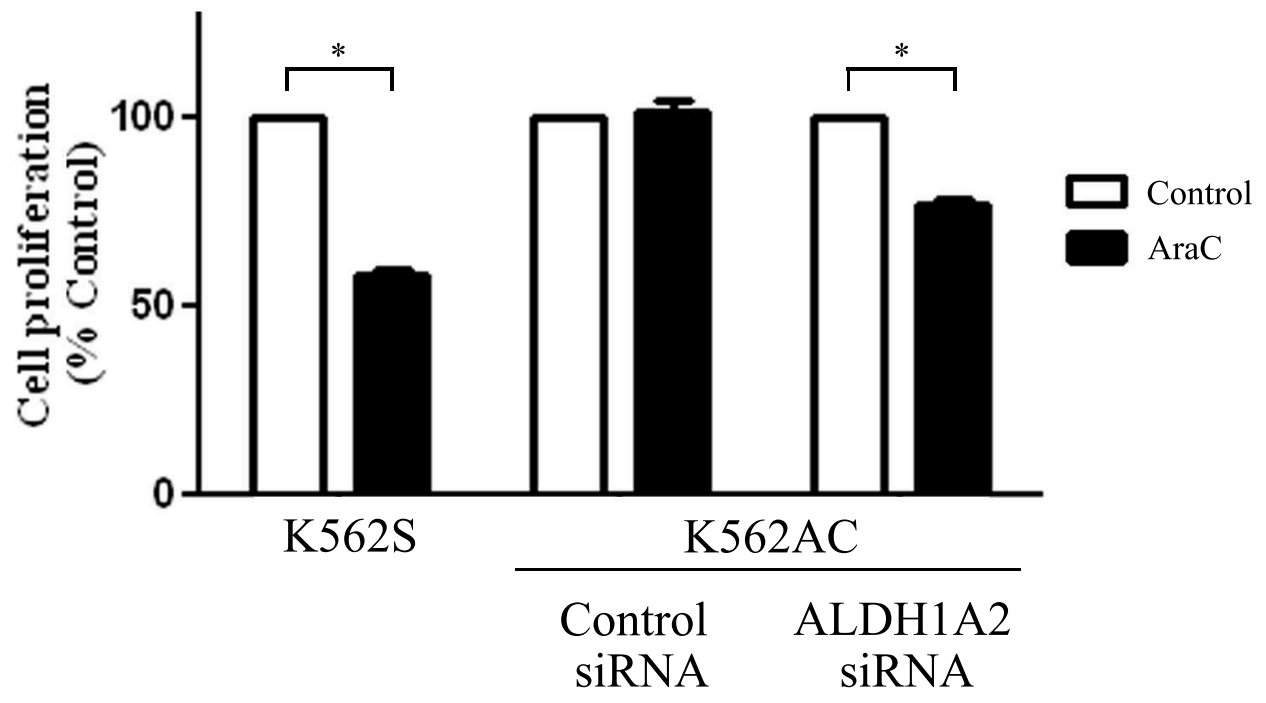

Figure 3. Kawasoe et al 
A

ALDH1A2

-ALDH1A2+GFP $\longleftarrow$ ALDH1A2

GAPDH

K562AC K562S

ALDH1A2

B

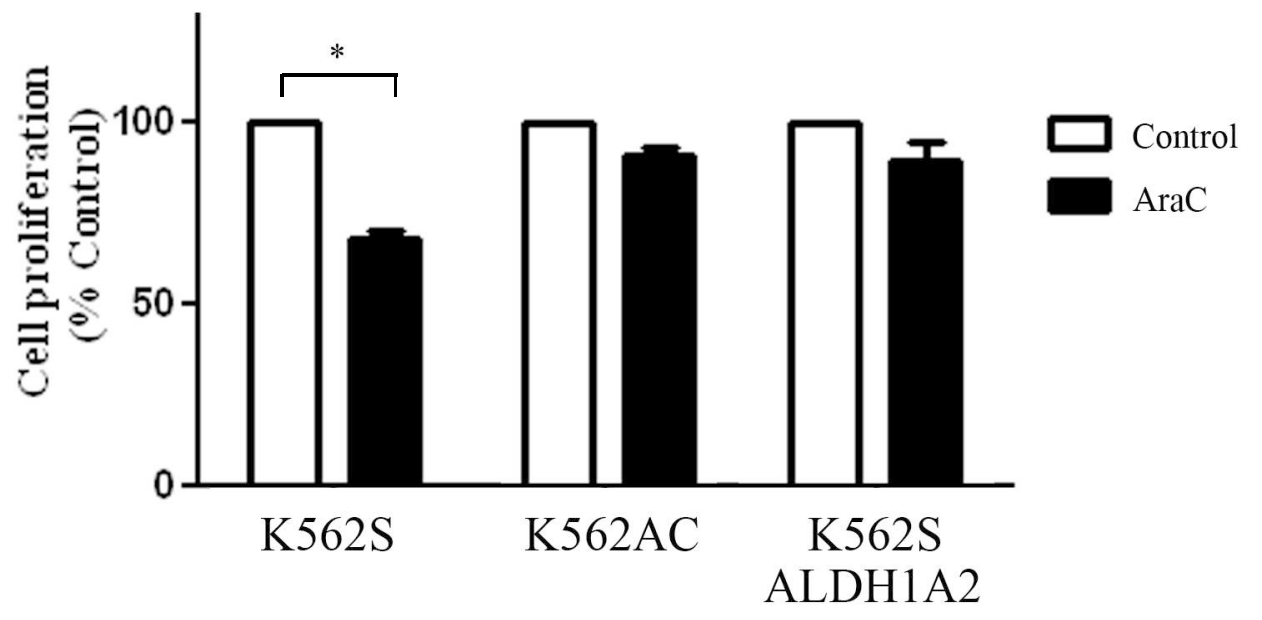

Figure 4. Kawasoe et al 
A

ALDH1A2
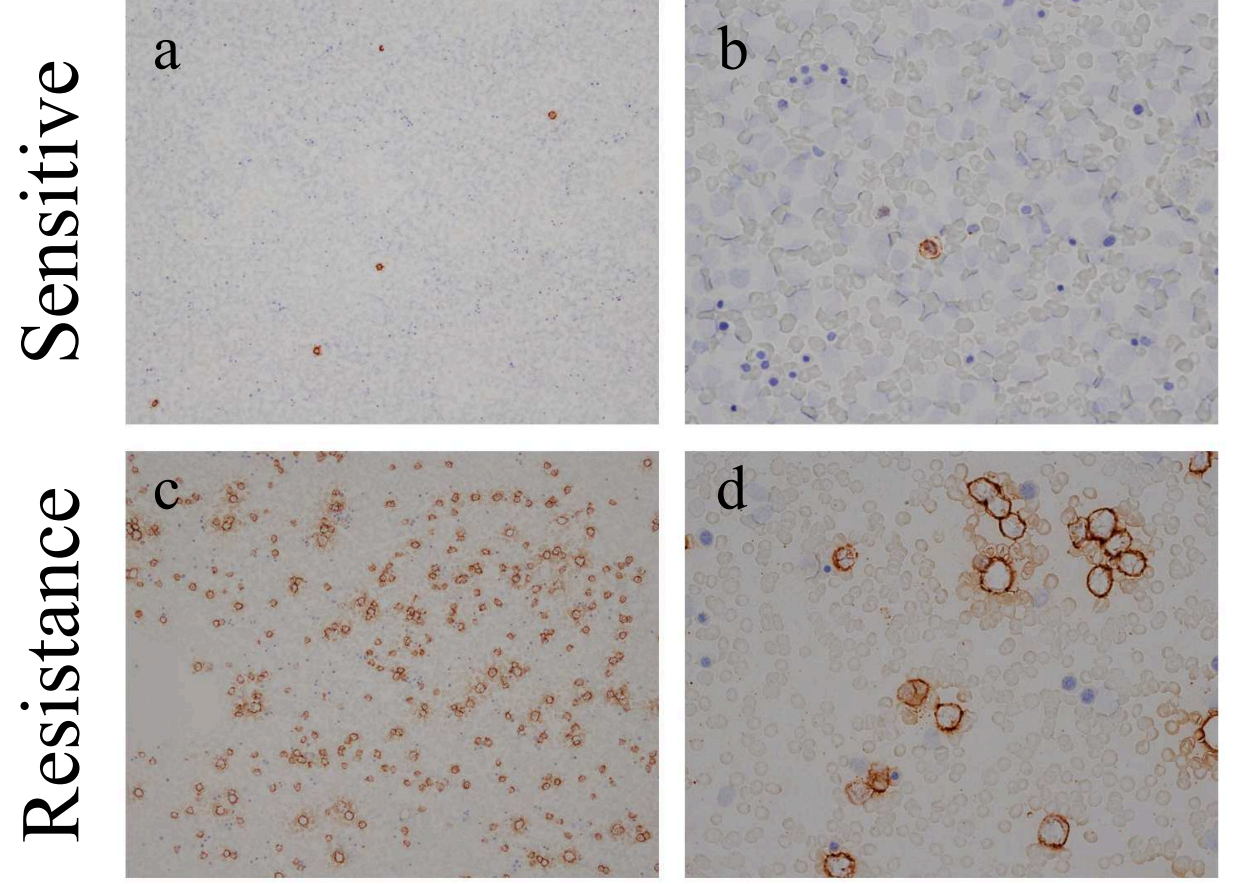

B

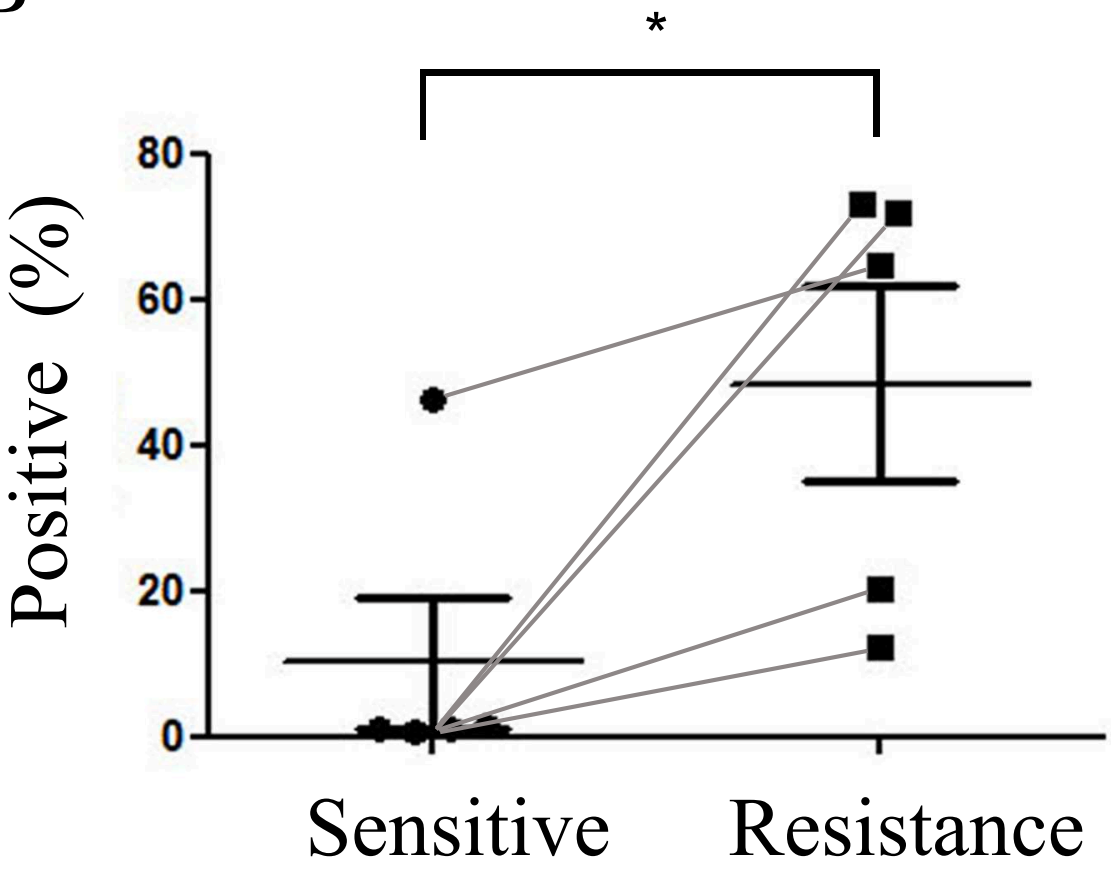

Figure 5. Kawasoe et al 
Table 1.

\begin{tabular}{cllcc}
\hline \multirow{2}{*}{ Spot no. Protein name } & & ID (NCBI) & p-value & Fold change \\
\hline 1 & Phosphoenolpyruvate carboxykinase & gi290457671 & 0.0016 & 2.18 \\
2 & Syntaxin-binding protein 1 & gi50403646 & 0.0016 & 2.53 \\
3 & Glucose-6-phosphate isomerase & gi17380385 & 0.0053 & -2.28 \\
4 & Aldehyde dehydrogenase 1 family, member A2 & gi25777724 & 0.00088 & 11.48 \\
5 & Vimentin & gi55977767 & 0.0016 & -3.59 \\
6 & D-3-phosphoglycerate dehydrogenase & gi21264510 & 0.0040 & 2.52 \\
7 & Cytokeratin-8 & gi90110027 & 0.00097 & 2.73 \\
8 & hnRNP D0 & gi13124489 & 0.0018 & -2.00 \\
9 & Ornithine aminotransferase & gi129018 & 0.00076 & -2.39 \\
10 & Medium-chain specific acyl-CoA dehydrogenase & gi113017 & 0.0023 & 2.63 \\
11 & Annexin A2 & gi113950 & 0.0049 & 2.00 \\
12 & Glutathione S-transferase omega-1 & gi6016173 & 0.00088 & 2.11 \\
13 & Carbonic anhydrase 2 & gi115456) & 0.0200 & -2.63 \\
14 & Phosphoglycerate mutase 1 & gi130348 & 0.0025 & 2.37 \\
15 & Supransgelin-2 & gi586000 & & -2.01 \\
\hline
\end{tabular}




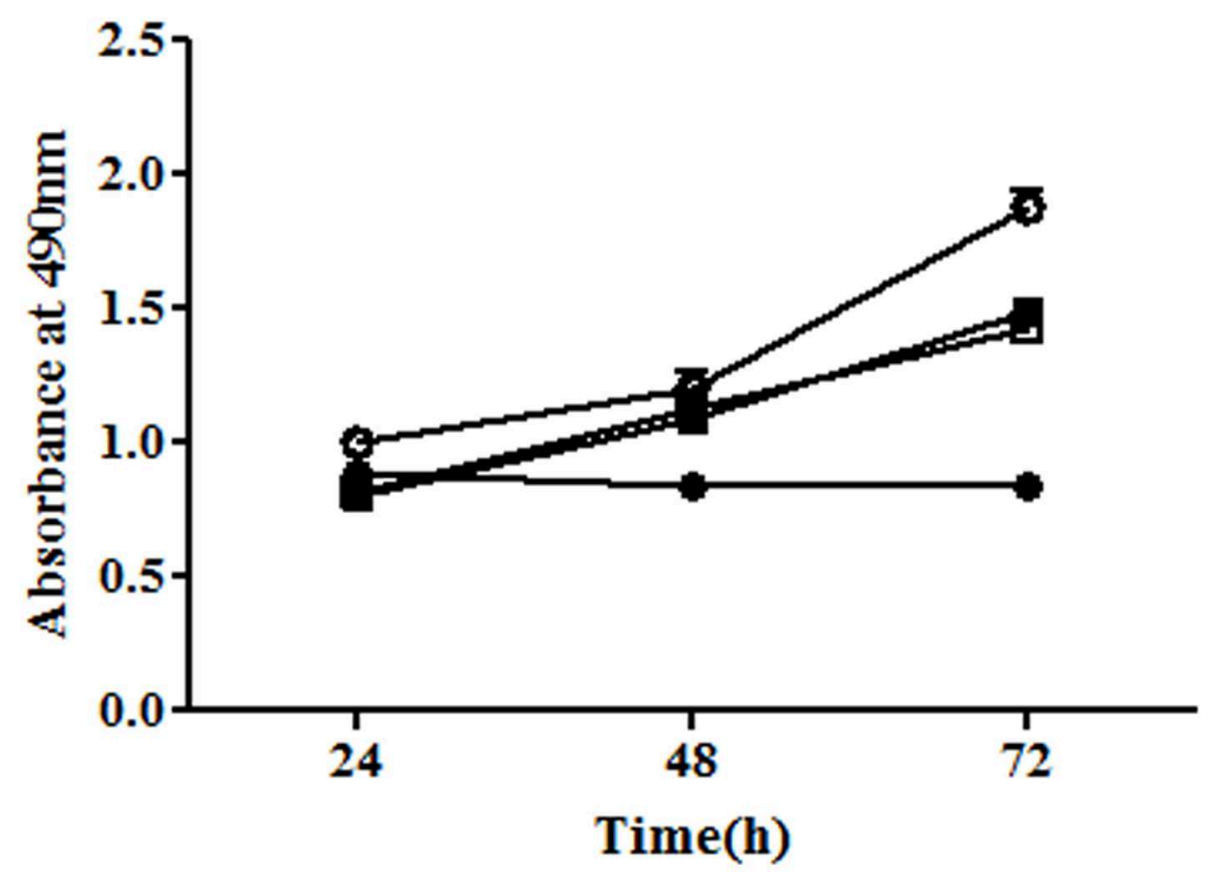

- K562S

- K562S Ara-C

Ð $\mathrm{K} 562 \mathrm{AC}$

$\rightarrow$ K562AC Ara-C

Time(h) 


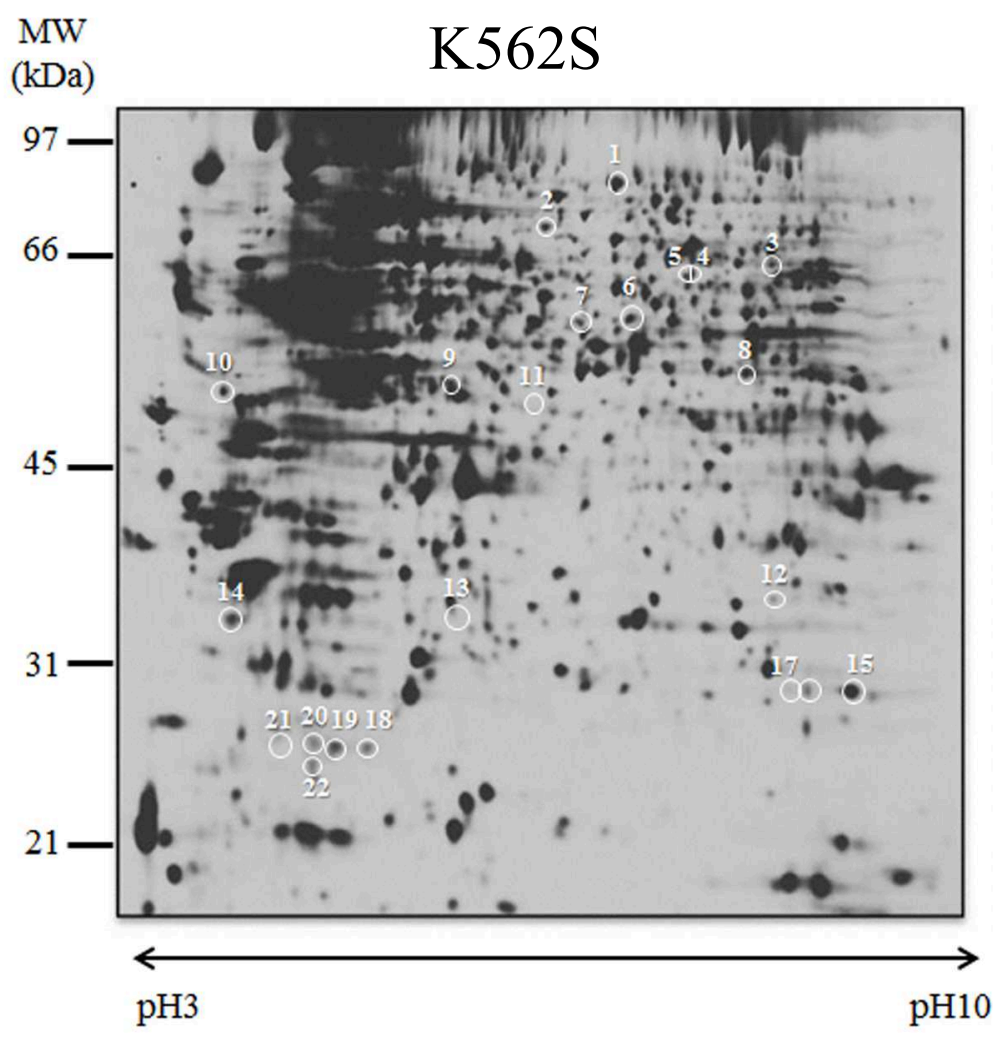

K562 doxorubicin

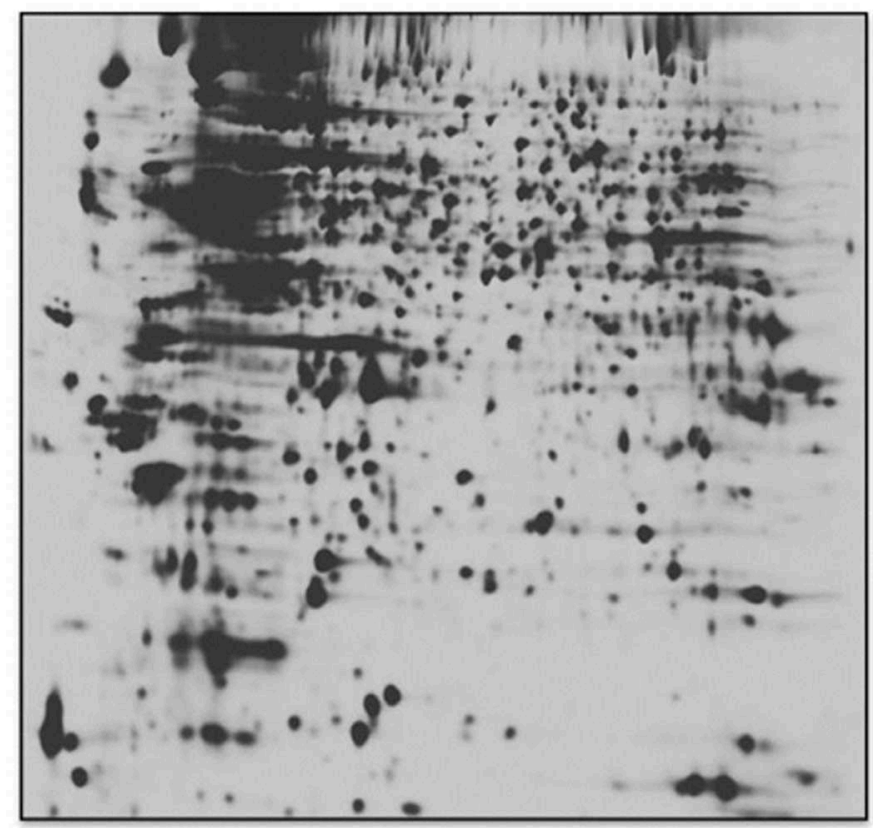

H10 


\section{Supplement Table 1}

\begin{tabular}{|c|c|c|c|}
\hline Characteristics of the patients & Age & Sex & FAB subtype \\
\hline Acute myelogenous leukemia & 59 & F & M2 \\
\hline & 31 & M & M4 \\
\hline & 37 & M & M4 \\
\hline & 48 & F & M5a \\
\hline & 53 & M & M5a \\
\hline
\end{tabular}


Supplemental table 2.

\begin{tabular}{|c|c|c|c|c|}
\hline Spot no. & Protein name & ID (NCBI) & p-value & Fold change \\
\hline 1 & Ezrin & gi125987826 & 0.0640 & 3.19 \\
\hline 2 & Leukotriene A-4 hydrolase & gi126353 & 0.0041 & 2.33 \\
\hline 3 & $\begin{array}{l}\text { Catalase } \\
\text { Pyruvate kinase isozymes R/L }\end{array}$ & $\begin{array}{l}\text { gi115702 } \\
\text { gi8247933 }\end{array}$ & 0.0054 & 2.31 \\
\hline 4 & Inosine-5'-monophosphate dehydrogenase 2 & gi124419 & 0.0021 & 2.92 \\
\hline 5 & Glucose-6-phosphate 1-dehydrogenase & gi116242483 & 0.0048 & 2.19 \\
\hline 6 & Lupus La protein & gi125985 & 0.0030 & 2.24 \\
\hline 7 & Lupus La protein & gi125985 & 0.0025 & 2.15 \\
\hline 8 & Multifunctional protein ADE2 & gi131628 & 0.0016 & 2.38 \\
\hline 9 & Serpin B9 & gi1709896 & 0.0020 & -2.39 \\
\hline 10 & Reticulocalbin-1 & gi2493462 & 0.0038 & -3.43 \\
\hline 11 & Macrophage-capping protein & gi313104088 & 0.0021 & 3.29 \\
\hline 12 & $\begin{array}{l}\text { Phosphoglycerate mutase } 1 \\
\text { Carbonic anhydrase } 2\end{array}$ & $\begin{array}{l}\text { gi1 } 130348 \\
\text { gi11 } 15456\end{array}$ & 0.0021 & -2.52 \\
\hline 13 & $\begin{array}{l}\text { 15-hydroxyprostaglandin dehydrogenase [NAD+] } \\
\text { Putative ATP-dependent Clp protease proteolytic subunit }\end{array}$ & $\begin{array}{l}\text { gi129889 } \\
\text { gi3023512 }\end{array}$ & 0.0050 & 5.61 \\
\hline 14 & $\begin{array}{l}\text { Kinetochore protein } \mathrm{Spc} 24 \\
\text { Calbindin }\end{array}$ & $\begin{array}{l}\text { gi74751176 } \\
\text { gi115394 }\end{array}$ & 0.0025 & -4.56 \\
\hline 15 & Peroxiredoxin-1 & gi548453 & 0.0021 & 2.30 \\
\hline 16 & Flavin reductase & gi1706870 & 0.0030 & 2.42 \\
\hline 17 & Flavin reductase & gi1706870 & 0.0024 & 2.60 \\
\hline 18 & Sorcin & gi267021 & 0.0048 & 4.60 \\
\hline 19 & Sorcin & gi267021 & 0.0066 & 2.12 \\
\hline 20 & Sorcin & gi267021 & 0.0018 & 6.05 \\
\hline 21 & Sorcin & gi267021 & 0.0025 & 2.81 \\
\hline 22 & Sorcin & gi267021 & 0.0021 & 3.72 \\
\hline
\end{tabular}

